8. Buckley JP, Sim J, Eston RG, Hession R, et al. Reliability and validity of measures taken during the Chester step test to predict aerobic power and to prescribe aerobic exercise. Br J Sports Med 2004;38(2):197-205.

9. De Andrade $\mathrm{CH}$, de Camargo AA, de Castro BP, Malaguti $\mathrm{C}$, et al.Comparison of cardiopulmonary responses during 2 incremental step tests in subjects with COPD. Respir Care 2012;57(11):1920-6.

10. Barkley JE, Roemmich JN. Validity of the CALER and OMNI-bike ratings of perceived exertion. Med Sci Sports Exerc 2008;40(4):760-6.
11. Groslambert A, Mahon AD. Perceived exertion: influence of age and cognitive development. Sports Med 2006;36(11): 911-28.

12. Zou GY. Sample size formulas for estimating intraclass correlation coefficients with precision and assurance. Stat Med 2012;31(29):3972-81.

13. Barroso R, Cardoso RK, do Carmo EC, Tricoli V. Perceived exertion in coaches and young swimmers with different training experience. Int J Sports Physiol Perform 2014;9(2): 212-6.

\title{
¿Está la transfusión de glóbulos rojos asociada a infecciones nosocomiales en niños críticamente enfermos?
}

\author{
Are red blood cell transfusions associated with nosocomial infections in critically \\ ill children?
}

\section{Dr. Omar E. Naveda Romero y Estud. Andrea F. Naveda Meléndeza}

\section{RESUMEN}

Aunque la transfusión de hemoderivados es una práctica común, los efectos sobre el sistema inmune no han sido bien estudiados. Para determinar la asociación entre transfusión de glóbulos rojos (TGR) e infecciones nosocomiales, se realizó un estudio decohorte prospectivocon niños críticamenteenfermos, seguidos hasta su fallecimiento, transferencia o egreso. Las infecciones nosocomiales se consideraron postransfusionales si ocurrieron dentro de los 14 días después de la TGR. Se incluyeron 162 niños, 35 adquirieron infección nosocomial $(21,6 \%)$ y 49 recibieron TGR $(30,2 \%)$. Los niños con infección nosocomial recibieron más frecuentemente TGR $(48,5 \%$ vs. 14,9\%; OR 5,4; IC 95\%: 2,4-12,6; $p<0,0001)$ y presentaron mayor mortalidad (45,7\% vs. $10,2 \%$; OR 7,4; IC 95\%: 3,1-18,2; $p$ $<0,0001)$. En la regresión logística binaria, la TGR se mantuvo asociada independientemente a infección nosocomial (OR 4,2; IC 95\%: 2,1-20,2; $p=0,049$ ).

Conclusión: La TGR se asoció a un incremento del riesgo de adquirir infecciones nosocomiales.

Palabras clave: transfusión sanguínea, glóbulos rojos, infecciones nosocomiales, niños.

\footnotetext{
a. Servicio de Trauma Shock y Estabilización. Hospital Universitario de Pediatría "Dr. Agustín Zubillaga". Barquisimeto. Estado Lara. Venezuela.
}

Correspondencia: Dr. Omar E. Naveda Romero, omarnavedamd@yahoo.com.

Financiamiento: Ninguno.

Conflicto de intereses: Ninguno que declarar.

Recibido: 6-9-2015

Aceptado: 28-1-2016 http://dx.doi.org/10.5546/aap.2016.347

\section{INTRODUCCIÓN}

La transfusión de glóbulos rojos (TGR) es una práctica común en la atención del niño críticamente enfermo. A pesar de la gran cantidad de hemoderivados que son administrados en las unidades de trauma, cirugía y cuidados intensivos pediátricos, los efectos de las transfusiones sanguíneas sobre el sistema inmune no han sido bien estudiados. ${ }^{1}$

Durante el último siglo, la TGR ha sido vista como un procedimiento simple que tiene un beneficio evidente. Sin embargo, esta práctica ha sido objeto de un mayor escrutinio. Se reconoce que hay riesgos en la TGR relacionados con el almacenamiento y sobre los efectos inmunomoduladores que se producen en casi todos los receptores, lo que resalta una posible asociación entre la TGR y un aumento de la mortalidad e infecciones intrahospitalarias. ${ }^{2,3,4}$

El reconocimiento de estos riesgos ha llevado a una postura más crítica frente a los beneficios asociados con la TGR. Los mecanismos subyacentes de la modulación de la respuesta inmune por la TGR pueden incluir modificadores biológicos de glóbulos blancos, alteración de las funciones de los linfocitos $T$, de las células natural killer y defectos en la presentación de antígenos. ${ }^{5}$

El objetivo fue determinar la posible asociación entre TGR e infecciones nosocomiales. 


\section{MATERIAL Y MÉTODO}

Se realizó un estudio de cohorte prospectivo, que incluyó pacientes menores de 15 años de edad, con afectación de uno o más sistemas corporales con riesgo de secuela funcional de gravedad o muerte, seguidos hasta su fallecimiento, transferencia o egreso, ingresados en el Hospital Universitario de Pediatría "Dr. Agustín Zubillaga" de la ciudad de Barquisimeto, Estado Lara, Venezuela, en los Servicios de atención combinada médica y quirúrgica entre el $1^{\circ}$ de febrero de 2012 y el $1^{\circ}$ de febrero de 2014. Fueron excluidos aquellos niños que fallecieron antes de las 24 horas de admisión, con sepsis o neumonía 48 horas antes de su ingreso o en las 48 horas posteriores a su hospitalización, con inmunodeficiencia demostrada, quemados, con catéteres de larga duración, aquellos con muestras de fluidos corporales consideradas contaminadas y los que recibieron quimioterapia antes o durante su hospitalización.

Todos los pacientes elegibles fueron monitorizados para evaluar el desarrollo de infección nosocomial. Las infecciones nosocomiales se consideraron postransfusionales si ocurrieron dentro de los 14 días posteriores a la TGR. En el grupo no expuesto a TGR, se registraron las infecciones nosocomiales que se presentaron durante la estadía hospitalaria. Las infecciones que ocurrieron 48 horas antes o dentro de las 48 horas del ingreso no se consideraron nosocomiales. La transfusión incluyó la recepción de uno o más empaques de concentrado globular no leucodepletado.

Para los sujetos no transfundidos, la hemoglobina basal fue la del ingreso; para los transfundidos, fue la hemoglobina anotada antes de la transfusión recibida. En los sujetos transfundidos más de una vez, la hemoglobina basal fue el promedio de las hemoglobinas antes de cada transfusión.

El programa utilizado para el análisis estadístico fue el SPSS versión 17.0. El U-test de Mann-Whitney y $t$ de Student fueron utilizados cuando se requirieron; las diferencias en distribuciones de variables categóricas fueron analizadas con $\mathrm{chi}^{2}$. Las variables relevantes del análisis univariable fueron utilizadas para crear un modelo de regresión logística con un valor de $p$ menor de 0,05 como criterio de entrada. Se usó un método de "introducir".

TABLA 1. Características de la población de estudio según la transfusión

\begin{tabular}{lcccc}
\hline & Todos & TGR & no TGR & $p$ \\
\hline Número de pacientes & 162 & 49 & 113 & --- - $^{*}$ \\
Edad (años) & $7,1 \pm 3,9$ & $9,1 \pm 4,1$ & $6,6 \pm 3,7$ & $0,001^{*}$ \\
Masculinos (\%) & $80(49,4)$ & $38(77,6)$ & $42(37,2)$ & $<0,0001$ \\
PRISM (puntos) & $13,9 \pm 3,2$ & $14,2 \pm 2,7$ & $14,1 \pm 0,4$ & $0,239^{*}$ \\
Días de hospitalización & $8,0 \pm 4,9$ & $10,5 \pm 2,3$ & $7,3 \pm 5,2$ & $<0,0001^{ \pm}$ \\
Ventilación mecánica (\%) & $90(55,6)$ & $40(81,6)$ & $50(44,2)$ & $<0,0001$ \\
Días de ventilación mecánica & $2,9 \pm 3,7$ & $6,3 \pm 1,1$ & $2,1 \pm 3,6$ & $<0,0001^{*}$ \\
Catéteres (\%) & $105(64,8)$ & $38(77,6)$ & $67(59,3)$ & 0,025 \\
Nutrición parenteral (\%) & $60(37,0)$ & $38(77,6)$ & $22(19,5)$ & $<0,0001$ \\
Infección nosocomial (\%) & $35(21,6)$ & $23(46,9)$ & $12(10,6)$ & $<0,0001$ \\
Infección del torrente sanguíneo (\%) & $15(9,2)$ & $9(18,4)$ & $6(5,3)$ & 0,008 \\
ITS (x 1000 días de CVC) & 14 & 15 & 12 & $0,061^{* *}$ \\
Sepsis (\%) & $10(6,2)$ & $8(16,3)$ & $2(1,8)$ & 0,0004 \\
Neumonía (\%) & $6(3,7)$ & $5(10,2)$ & $1(0,9)$ & 0,004 \\
Infección urinaria (\%) & $4(2,5)$ & $1(2,0)$ & $3(2,7)$ & 0,817 \\
Cirugía (\%) & $42(25,9)$ & $17(34,7)$ & $25(22,1)$ & 0,094 \\
Trauma (\%) & $27(16,7)$ & $8(16,3)$ & $19(16,8)$ & 0,939 \\
Complicaciones (\%) & $74(45,7)$ & $21(42,9)$ & $53(46,9)$ & 0,635 \\
Transfusión de plaquetas y/o PFC (\%) & $88(54,3)$ & $26(53,1)$ & $62(54,9)$ & 0,895 \\
Hemoglobina basal (g/dl) & $9,6 \pm 1,9$ & $8,4 \pm 0,6$ & $9,8 \pm 2,1$ & $<0,0001^{ \pm}$ \\
Mortalidad (\%) & $28(17,3)$ & $14(28,6)$ & $14(12,4)$ & 0,012 \\
\hline
\end{tabular}

${ }^{*} t$ de Student; ${ }^{* *}$ Estimado por regresión logística controlando días de procedimiento; ${ }^{£} \mathrm{U}$ de Mann-Whitney;

TGR: transfusión de glóbulos rojos; PRISM: riesgo pediátrico de mortalidad; ITS: infección del torrente sanguíneo;

CVC: catéter venoso central; VM: ventilación mecánica; PFC: plasma fresco congelado;

$\S$ Catéteres: incluyen percutáneos o por flebotomía, arteriales, drenaje peritoneal, ventriculostomía y derivación ventriculoperitoneal. 


\section{RESULTADOS}

Un total de 162 pacientes fueron incluidos en el estudio. 49 niños recibieron TGR $(30,2 \%)$ y 35 niños adquirieron infección nosocomial $(21,6 \%)$. Los datos de las características basales entre los niños transfundidos y no transfundidos están resumidos en la Tabla 1. De los 113 pacientes no transfundidos, 12 presentaron infección nosocomial; en los 49 niños transfundidos, 23 presentaron infección, lo que muestra una proporción significativamente mayor en este grupo ( $46,9 \%$ vs. $10,6 \% ; p<0,0001)$, en el que la infección del torrente sanguíneo $(18,4 \%$ vs. $5,3 \% ; p=0,008)$, sepsis $(16,3 \%$ vs. $1,8 \% ; p=0,0004)$ y la neumonía asociada al ventilador $(10,2 \%$ vs. $0,9 \% ; p=0,004)$ presentaron también una frecuencia significativamente mayor, al igual que la mortalidad $(28,6 \%$ vs. $12,4 \% ; p=0,012)$.

El análisis univariado de los factores de riesgo potenciales para infección nosocomial esta resumido en la Tabla 2. El grupo con infección nosocomial presentó una mayor estadía de hospitalización $(13,2 \pm 7,9$ vs. $6,7 \pm 2,5$ días; OR 1,3; IC 95\%: 1,2-1,5; $p<0,0001)$, con mayor proporción de ventilación mecánica $(82,9 \%$ vs. $48,8 \%$; OR 4,7 ; IC $95 \%: 1,8-12,2 ; p=0,001$ ) y mayor número de días de ventilación $(7,2 \pm 5,0$ vs. $1,9 \pm 2,2$ días; OR 1,6; IC 95\%: 1,3-1,8; $p<0,0001$ ). También los niños con infección nosocomial presentaron mayor proporción de catéteres $(82,9 \%$ vs. $60,6 \%$; OR 2,9 ; IC $95 \%$ : 1,2-7,6; $p=0,022$ ), nutrición parenteral $(80,0 \%$ vs. $26,0 \%$; OR 10,4 ; IC $95 \%$ : $4,1-26,1 ; p<0,0001)$, TGR $(48,5 \%$ vs. 14,9\%; OR 5,4; IC 95\%: 2,4-12,6; $p<0,0001)$, tres o más TGR (31,4\% vs. 5,5\%; OR 7,6; IC 95\%: 2,6$21,9 ; p<0,0001)$ y cirugía ( $42,9 \%$ vs. $22,0 \%$; OR 2,7 ; IC $95 \%: 1,2-6,0 ; p=0,015)$. La mortalidad también fue mayor ( $45,7 \%$ vs. $10,2 \%$; OR 7,4 ; IC $95 \%$ : $3,1-$ $18,2 ; p<0,0001)$.

En el análisis de regresión logística multivariado (Tabla 3), la TGR se mantuvo asociada independientemente a infección

TABLA 2. Análisis univariado: Factores de riesgo potenciales para infección nosocomial

\begin{tabular}{lcccc}
\hline & Infección nosocomial & sin infección nosocomial & OR (95\% IC) & $p$ \\
\hline Número de pacientes & 35 & 127 & & $\overline{0}$ \\
Edad (años) & $8,2 \pm 4,2$ & $6,8 \pm 3,8$ & $1,1(0,9-1,2)$ & $0,088^{*}$ \\
Masculinos (\%) & $17(48,5)$ & $63(49,6)$ & $1,0(0,4-2,1)$ & 0,908 \\
PRISM (puntos) & $15,6 \pm 3,0$ & $14,3 \pm 2,9$ & $0,9(0,3-1,8)$ & $0,137^{*}$ \\
Días de hospitalización & $13,2 \pm 7,9$ & $6,7 \pm 2,5$ & $1,3(1,2-1,5)$ & $<0,0001^{*}$ \\
Ventilación mecánica (\%) & $29(82,9)$ & $62(48,8)$ & $4,7(1,8-12,2)$ & 0,001 \\
Días de ventilación mecánica & $7,2 \pm 5,0$ & $1,9 \pm 2,2$ & $1,6(1,3-1,8)$ & $<0,0001^{*}$ \\
Catéteres (\%) & $29(82,9)$ & $77(60,6)$ & $2,9(1,2-7,6)$ & 0,022 \\
Nutrición parenteral (\%) & $28(80,0)$ & $33(26,0)$ & $10,4(4,1-26,1)$ & $<0,0001$ \\
TGR (\%) & $17(48,5)$ & $19(14,9)$ & $5,4(2,4-12,6)$ & $<0,0001$ \\
Tres o más TGR (\%) & $71(31,4)$ & $7,6(2,6-21,9)$ & $<0,0001$ \\
Cirugía (\%) & $15(42,9)$ & $28(22,0)$ & $2,7(1,2-6,0)$ & 0,015 \\
Trauma (\%) & $5(14,3)$ & $22(17,3)$ & $0,9(0,3-2,5)$ & 0,794 \\
Mortalidad (\%) & $13(10,2)$ & $7,4(3,1-18,2)$ & $<0,0001$ \\
\hline
\end{tabular}

* Regresión univariable; OR: odds ratio; IC: intervalo de confianza; TGR: transfusión de glóbulos rojos; PRISM: riesgo pediátrico de mortalidad.

Tabla 3. Análisis de regresión logística multivariado: evaluación del riesgo de infección nosocomial

\begin{tabular}{lcccc}
\hline Variable & $\beta$ & razón de odds & intervalo de confianza 95\% & $p$ \\
\hline Días de hospitalización & $-0,206$ & 0,8 & $0,6-1,1$ & 0,231 \\
Ventilación mecánica & 3,07 & 5,9 & $1,6-53,0$ & 0,023 \\
Días de ventilación mecánica & 1,05 & 2,8 & $1,4-5,9$ & 0,003 \\
Catéteres & 2,84 & 7,2 & $1,3-17,6$ & 0,038 \\
Nutrición parenteral & $-0,823$ & 0,5 & $0,1-7,4$ & 0,569 \\
TGR & 0,236 & 4,2 & $2,1-20,2$ & 0,049 \\
Tres o más TGR & $-0,341$ & 0,7 & $0,1-3,6$ & 0,169 \\
Cirugía & $-0,186$ & 0,8 & $0,2-2,9$ & 0,770 \\
\hline
\end{tabular}

TGR: transfusión de glóbulos rojos. $\beta$ : coeficiente $\beta$. El modelo clasifica correctamente al $93,2 \%$ de los casos.

Prueba de Hosmer y Lemeshow= 0,319. 
nosocomial (OR 4,2; IC 95\%: 2,1-20,2; $p=0,049$ ). Los otros factores asociados a infección nosocomial en la regresión fueron ventilación mecánica (OR 5,9; IC 95\%: 1,6-53,0; $p=0,023$ ), días de ventilación mecánica (OR 2,8; IC 95\%: 1,4-5,9; $p$ $=0,003)$ y presencia de catéteres (OR 7,2; IC 95\%: $1,3-17,6 ; p=0,038)$.

\section{DISCUSIÓN}

Las infecciones nosocomiales son un importante problema de salud pública. En la cohorte descrita, se ha identificado la TGR como un factor independiente asociado a infecciones nosocomiales en áreas de atención médica / quirúrgica del niño críticamente enfermo. De acuerdo con el análisis de regresión logística, los factores independientes asociados a un incremento del riesgo para infecciones nosocomiales fueron ventilación mecánica, días de ventilación mecánica, la presencia de catéteres y transfusión de glóbulos rojos. Datos similares son reportados en la literatura ${ }^{6,7,8}$

La ventilación mecánica y la presencia de catéteres invasivos son factores de riesgo conocidos de infecciones nosocomiales. ${ }^{9,10} \mathrm{La}$ asociación entre TGR e infección nosocomial es biológicamente posible a través de mecanismos conocidos. ${ }^{11,12 \text {, }}$ ${ }^{13}$ Las transfusiones pueden ser inherentemente inmunosupresoras. Los mecanismos propuestos para esta inmunosupresión incluyen la inducción de supresión de células $\mathrm{T}$ y la disminución de la actividad de los linfocitos $\mathrm{T}$ natural killer. ${ }^{14}$ También se ha documentado una disminución en la producción de interleucina- 2 y un aumento en la producción de prostaglandina E2, con una disminución en los linfocitos T CD4 helper y del receptor positivo para la interleucina- 2 en células auxiliares. Sin embargo, el interés reciente se ha centrado en los efectos inmunomoduladores y en las lesiones por almacenamiento de hematíes transfundidos..$^{15}$

Un aspecto para tomar en cuenta se refiere a la cuestión de si la asociación observada entre TGR e infección nosocomial representa una asociación real o una relación casual dada por la mayor gravedad de la enfermedad en los pacientes que requieren transfusiones. El riesgo pediátrico de mortalidad (pediatric risk of mortality; PRISM, por sus siglas en inglés) no se estratificó en la investigación; sin embargo, se observó que los niños transfundidos o no presentaron una puntuación del PRISM similar, al igual que los niños con o sin infección nosocomial. Además, la proporción de complicaciones y agravamientos resultó similar en ambos grupos. Se podría inferir que la gravedad de la enfermedad no constituyó un factor de riesgo asociado en la serie estudiada, a pesar de que el grupo transfundido presentó mayor tiempo de hospitalización y, por ende, de exposición a dispositivos.

Otra limitación estuvo dada por la carencia de un evento que permitiera tener un límite a partir del cual se realizaran las observaciones de infecciones en la cohorte no expuesta. Además, no se tomaron en cuenta otras neumonías nosocomiales en sujetos no ventilados mecánicamente.

\section{CONCLUSIÓN}

Los supuestos de que la TGR proporciona un tratamiento efectivo para la anemia con riesgos mínimos en niños críticamente enfermos han sido puestos en duda. La asociación entre la TGR e infecciones nosocomiales descrita en esta cohorte siempre será vulnerable a la crítica de que tal asociación refleja la tendencia de los médicos a transfundir a los pacientes más graves. Los datos apuntan a la probabilidad de que la TGR sea un importante contribuyente en la presentación de infecciones nosocomiales en niños críticamente enfermos.

\section{REFERENCIAS}

1. White M, Barron J, Gornbein J, Lin JA. Are red blood cell transfusions associated with nosocomial infections in pediatric intensive care units? Pediatr Crit Care Med 2010;11(4):464-8

2. Istaphanous GK, Wheeler DS, Lisco SJ, Shander A. Red blood cell transfusion in critically ill children: a narrative review. Pediatr Crit Care Med 2011;12(2):174-83.

3. Armano R, Gauvin F, Ducruet T, Lacroix J. Determinants of red blood cell transfusions in a pediatric critical care unit: a prospective, descriptive epidemiological study. Crit Care Med 2005;33(11):2637-44.

4. Shorr AF, Jackson WL, Kelly KM, Fu M, et al. Transfusion practice and blood stream infections in critically ill patients. Chest 2005;127(5):1722-8.

5. HoJ, Sibbald WJ, Chin-YeeIH. Effects of storage on efficacy of red cell transfusion: when is it not safe? Crit Care Med 2003;31(12 Suppl):S687-97.

6. Raghavan M, Marik PE. Anemia, allogenic blood transfusion, and immunomodulation in the critically ill. Chest 2005;127(1):295-307.

7. Vamvakas EC, Blajchman MA. Transfusion-related immunomodulation (TRIM): an update. Blood Rev 2007;21(6):327-48.

8. Jeschke MG, Chinkes DL, Finnerty CC, Przkora R, et al. Blood transfusions are associated with increased risk for development of sepsis in severely burned pediatric patients. Crit Care Med 2007;35(2):579-83.

9. Díaz E, Lorente L, Valles J, Rello J. Neumonía asociada a la ventilación mecánica. Med Intensiva 2010;34(5):318-24.

10. Pronovost $\mathrm{P}$, Needham D, Berenholtz S, Sinopoli D, et al. An intervention to decrease catheter-related bloodstream infections in the ICU. N Engl J Med 2006;355(26):2725-32. 
11. Blumberg N. Deleterious clinical effects of transfusion immunomodulation: proven beyond a reasonable doubt. Transfusion 2005;45(2 Suppl):33S-40S.

12. Silverboard H, Aisiku I, Martin GS, Adams M, et al. The role of acute blood transfusion in the development of acute respiratory distress syndrome in patients with severe trauma. J Trauma 2005;59(3):717-23.

13. Shorr AF, Duh MS, Kelly KM, Kollef MH. Red blood cell transfusion and ventilator-associated pneumonia: a potential link? Crit Care Med 2004;32(3):666-74.
14. Bateman ST, Lacroix J, Boven K, Forbes P, et al. Anemia, blood loss, and blood transfusions in North American children in the intensive care unit. Am J Respir Crit Care Med 2008;178(1):26-33.

15. Valentine SL, Lightdale JR, Tran CM, Jiang H, et al. Assessment of hemoglobin threshold for packed RBC transfusion in a medical-surgical PICU. Pediatr Crit Care Med 2014;15(2):e89-94.

\title{
Publicación de trabajos presentados en los Encuentros Nacionales de Investigación Pediátrica de la Sociedad Argentina de Pediatría: factores involucrados
}

\author{
Publication of abstracts presented at the National Pediatric Research Meetings \\ of the Argentine Society of Pediatrics: Related factors
}

\author{
Dra. Paula Dominguez ${ }^{a}$ Dra. María F. Ossorio ${ }^{a, b}$, Dr. Eduardo Cuestas ${ }^{b, c}$, Dr. Norberto Giglio ${ }^{b, d}$, \\ Dr. Carlos Grandib, , Dr. Facundo García-Bournissen ${ }^{b, d}$, Dr. Santiago Vidaurreta ${ }^{b, f}$, Dr. Jaime Altcheh ${ }^{b, d}$ y \\ Dr. Fernando Ferrero ${ }^{a, b}$
}

\begin{abstract}
RESUMEN
Objetivos: Estimar la proporción de trabajos presentados en Encuentros Nacionales de Investigación Pediátrica que posteriormentefueron publicados en forma completa; describir sus diseños y factores involucrados con la no publicación. Métodos: Estudio descriptivo y analítico, que incluyó todos los trabajos presentados en Encuentros Nacionales de Investigación Pediátrica (1998-2011). Seidentificó un investigador por estudio y se lo encuestó sobre el diseño, la publicación y los factores asociados a la no publicación.

Resultados: Sobre 746 trabajos presentados, respondieron la encuesta los autores de 522 (70\%). El 84,3\% eran estudios observacionales y $15,7 \%$, experimentales; $34 \%$ recibieron financiación. Fueron publicados posteriormente 217 trabajos (41,5\%, IC 95\%: 37,3-45,9). Aquellos con financiación tuvieron
\end{abstract}

a. Hospital General de Niños Pedro de Elizalde, Buenos Aires.

b. Subcomisión de Investigación Pediátrica, Sociedad Argentina de Pediatría.

c. Hospital Privado de Córdoba, Córdoba.

d. Hospital General de Niños Ricardo Gutiérrez, Buenos Aires.

e. Hospital Materno-infantil Ramón Sardá, Buenos Aires.

f. Hospital Universitario CEMIC, Buenos Aires.

Correspondencia: Dra. Paula Domínguez, pau.dom@gmail.com

Financiamiento: La Dra. Paula Domínguez contó con una beca de investigación de la Sociedad Argentina de Pediatría para el desarrollo del estudio.

Conflicto de intereses: Ninguno que declarar.

Recibido: 8-12-2015

Aceptado: 3-2-2016 mayor probabilidad de publicarse (OR 2; IC 95\%: 1,4-2,9; $\mathrm{p}<0,001)$. Las causas de no publicación más frecuentes fueron la falta de tiempo, el tamaño muestral insuficiente y la dificultad con el financiamiento.

Conclusión: El 41,5\% de trabajos presentados en Encuentros Nacionales de Investigación Pediátrica alcanzaron la publicación en texto completo. La falta de tiempo fuela principal causa para no hacerlo.

Palabras clave: bibliografía de medicina, publicaciones periódicas, informe de investigación.

http:/ /dx.doi.org/10.5546/aap.2016.351

\section{INTRODUCCIÓN}

Pocas instituciones se han dedicado más a la salud infantil que la Sociedad Argentina de Pediatría (SAP). Algunas iniciativas pueden mostrar un impacto fácilmente identificable (p.e.: iniciativa sobre retinopatía del prematuro); en otros casos, aun cuando tengan un impacto importante, los resultados no suelen ser tan fáciles de identificar (p.e.: actividades de educación continua). Finalmente, las actividades de investigación promovidas por la SAP, que estimulan el espíritu crítico en los profesionales y generan conocimiento original, permitirán diseñar y/o validar herramientas y estrategias que colaborarán en forma directa con la prevención de la morbimortalidad infantil. En esta iniciativa, se incluyen los Encuentros Nacionales de Investigación Pediátrica que la SAP 